\title{
Stress-induced memory enhancement for inhibitory fear conditioning in rats
}

\author{
ROBERT W. FLINT, JR., MITCHELL M. METZGER, DON M. BENSON, JR., and DAVID C. RICCIO \\ Kent State University, Kent, Ohio
}

\begin{abstract}
The retroactive effects of stress on memory have not received a great deal of empirical attention; however, the research that has been conducted has reported both positive and negative effects of stress on memorial processes. The goal of this study was to examine the effects of a naturalistic stressoran intense bout of exercise-on memory for inhibitory fear conditioning in rats. Experiment 1 investigated the retroactive effect of a stressful swim treatment on memory for passive avoidance (PA) training. Rats that received an immediate posttraining swim treatment demonstrated a significant enhancement in performance when tested for retention $24 \mathrm{~h}$ later. Furthermore, the enhancing effect of the swim treatment was time dependent: Rats receiving the swim treatment 15 min after PA training no longer exhibited reliably better scores than did rats not receiving the swim treatment. Experiment 2 used preexposures to control for the possibility that the swim treatment was enhancing avoidance scores by acting as a punisher rather than a memory modulator. Results indicate that both the group that was preexposed and the group that was not preexposed showed reliably higher scores than did a group of animals receiving only PA training, thus replicating Experiment 1. Furthermore, the results of Experiment 2 suggest that swimming was not simply acting as a punishing agent, since preexposures to the treatment did not attenuate its memory-enhancing properties. The possible role of stress-related hormones on memory processes is considered.
\end{abstract}

The varied effects of a stressful experience on an organism have received a great deal of attention in both the biomedical and psychological fields. The various reports using the term stress refer to a wide range of stimulus conditions, including stress as a stimulus condition, stress as a response to stimulus conditions, or stress as a relationship involving stimuli, organism characteristics, and responses (Newberry, Jaikins-Madden, \& Gerstenberger, 1991). For clarity, we will refer to stress as a response to stimulus conditions, since our measure of stress is a state of fatigue resulting from intensive exercise.

Several reports have suggested that stressful experiences may enhance memory for certain learning tasks. White and LeGree (1984) reported that rats that were administered a single footshock immediately following conditioned emotional response (CER) training demonstrated significantly better retention of the CER training than did rats that did not receive the posttraining stressor. A similar enhancement of learning with posttraining admini-

\footnotetext{
The research reported here was supported in part by National Institute of Mental Health Grant MH37535 to D.C.R. Care of animals was in accordance with Kent State University's Animal Care and Use Committee guidelines. We thank B. H. Newberry for comments on an earlier draft of this manuscript. Portions of this study were presented at the 67th annual meeting of the Midwestern Psychological Association, Chicago, May 1995. D.M.B., Jr., is now at Northeastern Ohio Universities College of Medicine, Rootstown, $\mathrm{OH}$. Correspondence should be addressed to R. W. Flint, Jr., Department of Psychology, Kent State University, Kent, OH 44242-0001 (e-mail: rflint@kentvm. kent.edu).
}

stration of a stressful experience has also been reported for rats that were conditioned in a T-maze with an aversive footshock (Kumar \& Karanth, 1993). Following conditioning, animals were administered escapable, inescapable, or no-shock stress treatments. The results of this study indicate that the group of rats subjected to inescapable shock after the training session demonstrated enhanced avoidance to the previously shocked goalbox. Additionally, exposure to stressful situations prior to a learning episode has been demonstrated to facilitate the expression of a learned behavior. It has been reported that rats exposed to inescapable low-intensity tail shock prior to conditioning of an eye-blink response demonstrated significantly more eye-blink responses over 4 days of conditioning (Shors, Weiss, \& Thompson, 1992). Taken together, these findings suggest that the administration of stressful experiences either before or after training can enhance memory for the training episode.

In each of the studies discussed above, shock, which is a unique form of punishment involving an acute painful component, was used as the stressor. One question that arises is whether a stressor that is relatively devoid of acute painful features, such as intensive exercise, would also enhance memory for aversively motivated learning.

One plausible explanation for the enhancing effect of stressors is the chemical and/or hormonal changes in the body that accompany a stressful experience. Dimsdale, Hartley, Guiney, Ruskin, and Greenblat (1984) have presented evidence that levels of epinephrine released from the adrenal medulla may as much as triple during intensive exercise. Furthermore, Ellis, Kennedy, Eusebi, and Vincent (1967) reported that increases in endogenous 
blood epinephrine stimulate the release of glucose. On the behavioral level, an impressive array of data has been compiled suggesting that immediate posttraining injections of epinephrine or glucose improve performance on memory tasks in rodents and humans (Gold, 1991; McGaugh, 1989). Finally, in addition to increasing blood glucose levels and enhancing memory, epinephrine also stimulates an increase in the release of adrenocorticotropic hormone (ACTH; Axelrod \& Reisine, 1984). ACTH has also been implicated in memory processes: Research has shown that administration of ACTH facilitates learning and memory in both humans and animals (de Weid, 1980; Richardson, Riccio, \& Devine, 1984). Thus, it is plausible that facilitated performance in subjects receiving stress treatment may be a function of changes in endogenous glucose, epinephrine, and/or ACTH levels.

In the present study, Experiment 1 addressed whether an immediate posttraining swim treatment would improve memory for passive avoidance (PA). On the basis of the significant effect of swim treatment on memory modulation in Experiment 1, Experiment 2 was designed to control for the possibility of additive effects of the swim treatment with the footshock from training. If the swim treatment combined with the footshock to create a more punishing unconditioned stimulus (UCS), preexposures to the swim treatment prior to conditioning might attenuate its effectiveness as a punisher.

\section{EXPERIMENT 1}

Previous research has demonstrated that an acute exposure to a stressor such as footshock, separate from and following CER training, results in an enhanced level of performance in animals tested for fear retention $24 \mathrm{~h}$ later (White \& LeGree, 1984). The present experiment was designed to examine whether forced swimming, a stressor that does not appear to involve acute painful stimulation, would yield similar memory-facilitating effects if administered immediately after PA training.

\section{Method}

Subjects. Forty naive, male, Long-Evans rats served as subjects. All animals were obtained from the Kent State University breeding colony and were between 60 and 80 days of age, with weights ranging from 190 to $350 \mathrm{~g}$ at the time of training. Animals were housed individually in hanging wire mesh cages in a colony room maintained on a 15:09-h light:dark cycle and a constant temperature of approximately $70^{\circ} \mathrm{F}$. All experimentation took place during the light portion of the light:dark cycle, and food and water were available ad libitum throughout the experiment.

Apparatus. A standard PA apparatus was used in both experiments. The apparatus measured $33 \times 17 \times 17 \mathrm{~cm}$, was constructed of Plexiglas and steel, and was divided into two equal-sized compartments with an $8 \times 10 \mathrm{~cm}$ guillotine door separating the two chambers. One chamber was made of white Plexiglas with a clear Plexiglas lid, and the other chamber was constructed entirely of black Plexiglas. The floor of the apparatus was constructed of stainless steel bars $2 \mathrm{~mm}$ in diameter and spaced $1 \mathrm{~cm}$ apart. The black compartment was equipped to administer a 2 -sec, $120-\mathrm{V}$ footshock from a matched-impedance $\mathrm{AC}$ shock source. The experimental room was illuminated by a $15-\mathrm{W}$ light bulb that was suspended $30 \mathrm{~cm}$ above the white compartment. Furthermore, all experimentation was conducted in the presence of white noise to mask any background disturbances. Stopwatches were used to measure each subject's initial crossover latency and total time spent in the white compartment (TTW) as dependent measures.

The swim tank was located in a separate room from the training apparatus with different lighting and contextual surroundings. The tank was a rectangular Rubbermaid trash can with inside dimensions of $30 \times 30 \times 58 \mathrm{~cm}$. The tank was filled with room temperature water (approximately $18^{\circ} \mathrm{C}$ ) up to $10 \mathrm{~cm}$ from the top. Each subject that received the swim treatment had a $10 \mathrm{~g}$ weight attached to the base of its tail to force it to swim rather than to simply float.

Procedure. Subjects were randomly divided into five groups of 8 animals each, and all were trained individually in the PA apparatus. Each subject was brought into the experimental room and was allowed to observe the contextual cues present in the room for a 10 -sec period before being placed in the white chamber facing away from the guillotine door. After $10 \mathrm{sec}$, the door was lifted, allowing access to the black chamber. Once the subject had crossed into the black chamber (four-paw criterion), the door was immediately shut and a 2-sec, $120-\mathrm{V}$ inescapable footshock was delivered to each animal in the following groups: PA only, PA followed immediately by swim treatment (IMM), and PA followed by a 15min delay in the home cage before the swim treatment (delay). In the remaining two groups, control and swim, each subject was removed from the black chamber approximately $2 \mathrm{sec}$ after crossing over from the white side, and no shock was administered. The initial crossover latency for each subject was recorded to the nearest second with a stopwatch.

Following training, animals in the PA and control groups were returned to their home cages. The swim and IMM groups were immediately administered the swim treatment and subjects in the delay group were placed in their home cages for $15 \mathrm{~min}$ before the swim treatment was administered.

The swim treatment involved attaching a 10 -g weight to the animal's tail, approximately $2.5 \mathrm{~cm}$ from the base with a piece of insulated wire, and placing the subject in the swim tank. Animals were required to swim to a criterion of fatigue, which was specified as 15 consecutive sec without surfacing. After reaching the criterion, subjects were removed from the swim tank, towel dried, returned to their home cages, and the total amount of time spent swimming in the tank was recorded.

The test for retention of PA training was conducted approximately $24 \mathrm{~h}$ after conditioning. Subjects were returned to the white chamber of the apparatus facing away from the guillotine door for a $600-\mathrm{sec}$ retention test. After $10 \mathrm{sec}$, the door was lifted and the crossover latency and TTW scores were recorded to the nearest second with a stopwatch. If an animal failed to cross to the black side within $10 \mathrm{~min}$, it was arbitrarily assigned a score of $600 \mathrm{sec}$ for both latency and TTW. No shock was administered during the retention test

The intensity and duration of footshock used in Experiment 1 were based on pilot research indicating that a $2-\mathrm{sec}, 120-\mathrm{V}$ footshock was insufficient to induce ceiling-level scores on a $24-\mathrm{h}$ retention test. This was an important requirement for the training procedure since we were attempting to examine the effects of the stressor on memory modulation.

\section{Results and Discussion}

Initial crossover latencies were compared using a Kruskal-Wallis analysis of variance (ANOVA), which revealed that there were no reliable differences among any of the groups on the training day $(H=5.38, p>.05)$. A subsequent Kruskal-Wallis ANOVA computed for crossover latencies on the retention test also failed to re- 
veal any significant differences among the groups ( $H=$ $4.23, p>.05$ ); however, the same test on TTW scores indicated that there were reliable differences among the groups on the preference measure $(H=15.05, p<.05)$. Figure 1 presents the median TTW scores for all the groups on the 24-h retention test.

As can be seen from Figure 1, the IMM group (which received an immediate posttraining swim treatment) demonstrated a higher preference for the safe chamber of the PA apparatus than did the PA group $(U=4, p<.01)$. However, the effectiveness of the swim treatment to enhance memory was time dependent: If the swim treatment was delayed for $15 \mathrm{~min}$, there was no apparent memory facilitation (delay vs. PA; $U=24, p>.10$ ). This finding suggests that the stressor must occur relatively soon after training to exert a memory-enhancing effect.

One interpretive question is whether the IMM group had better retention scores on the PA task because the swim treatment merely acted as a punisher subsequent to the footshock. If the swimming treatment functioned as a UCS, subjects in the IMM group may have received two punishers contingent on entering the black chamber (i.e., footshock and swim treatment). Furthermore, the delay condition does not control for this additive effect, since a delay in punishment would also be less effective. However, a comparison between the control group (which did not receive shock or swim treatment) and the swim group (which did not receive footshock) failed to reveal any reliable differences between the groups $(U=31, p>$ .05 ). Thus, the swim treatment was not so aversive that it would act as punishment for animals not receiving footshock in the black chamber. Although this comparison does not rule out the possibility of additive effects between the footshock and swim treatment, it makes a double-punishment interpretation less plausible.
One discrepancy in Experiment 1 was that the effect for TTW scores was significant and reliable, whereas the crossover latencies failed to reach significance. Total time on white, rather than latency scores, has been reported as possibly being a more sensitive measure of fear retention in PA conditioning (Flint \& Riccio, 1996; Harrod, Metzger, \& Riccio, 1996). Occasionally, subjects will cross to the black (shock) side and then quickly return to the white (safe) chamber, resulting in short latencies and long TTW scores. This may account for why we did not obtain significant results between groups on the latency measure, but did obtain reliable differences between the groups on the preference measure.

In summary, it appears that an immediate, posttraining swim treatment can act to enhance performance of memory for one-trial inhibitory conditioning in rats. However, because of the nature of the paradigm, the possibility remains that the swim treatment enhanced avoidance to the black compartment simply because it acted as a punisher rather than a memory modulator. The aim of Experiment 2 was to address this alternative explanation.

\section{EXPERIMENT 2}

Despite the finding that the control groups did not reliably differ from each other in Experiment 1, the possibility remains that the swim treatment was acting as an additional punisher in conjunction with the footshock to produce higher retention scores in the IMM group. Experiment 2 sought to address this possibility by taking advantage of the finding that shock preexposures significantly decrease the effectiveness of shock in a punishment or conditioned-suppression paradigm (Capaldi, Sheffer, Viveiros, Davidson, \& Campbell, 1985; Randich, 1981). If the stress treatment is acting as an additional UCS, its

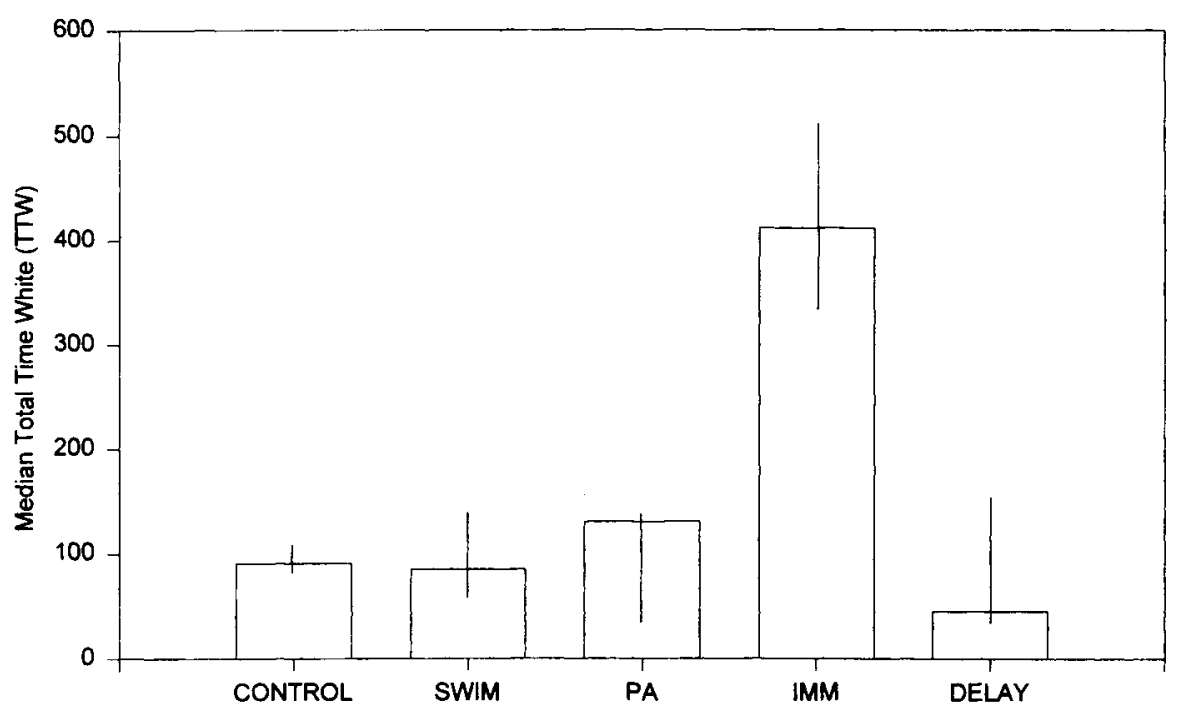

Figure 1. Median TTW scores from the 600-sec retention test for the control, swim, PA, IMM, and delay groups in Experiment 1. (Error bars = semi-interquartile range.) TTW, total time spent in the white compartment; PA, passive avoidance; IMM, PA followed immediately by swim treatment. 
effectiveness should also be attenuated with preexposures (i.e., UCS preexposure effect). Furthermore, in this experiment, preexposures to the swim treatment were administered in a context different from that of PA training. With this procedure, the preexposures may become associated with the context in which they occurred, making the preexposure context resistant to the development of any association with the PA training context. In summary, if the swim treatment is having a modulating effect on the memory for PA training, we would anticipate no difference between the group that received multiple pretraining exposures and the group that received only a single posttraining exposure.

\section{Method}

Subjects. Twenty-four naive, male, Long-Evans rats served as subjects. The animals were between 60 and 80 days of age and their weights ranged from 260 to $370 \mathrm{~g}$ at the time of training. All subjects were obtained from the Kent State University breeding colony and maintained under the same conditions as those outlined in Experiment 1. As in Experiment 1, all manipulations occurred during the light portion of the light:dark cycle.

Apparatus. The same PA apparatus and swim tank as those used in Experiment 1 were used in the present experiment.

Procedure. All animals were randomly divided into three groups of 8 animals each. The preexposed (PRE) group received a single swim treatment each day for 7 consecutive days prior to training (as described in Experiment 1), whereas animals in the no-PRE and PA groups were left undisturbed in their home cages during the preexposure phase. The swim treatment criterion was maintained each day during preexposures in order to maintain a controlled level of treatment among animals in the PRE group and to control for any tolerance to the swim treatment. On the training day, the animals in groups PRE and no-PRE were administered PA training (identical to that described earlier) and were then immediately given the swim treatment after removal from the apparatus. Animals in the PA group were simply returned to their home cages immediately after PA training.
Each animal was administered a $600-\mathrm{sec}$ retention test for fear to the black compartment $24 \mathrm{~h}$ after training. If an animal failed to cross to the black chamber within $10 \mathrm{~min}$, it was arbitrarily assigned a score of 600 -sec for latency and TTW. No shock was administered during the retention test.

\section{Results and Discussion}

As in Experiment 1, a Kruskal-Wallis ANOVA computed on initial crossover latencies for the training day did not yield any significant differences among the groups $(H=4.01, p>.05)$. Furthermore, the same pattern of results for retention scores that was obtained in Experiment 1 was seen in this experiment. The Kruskal-Wallis ANOVA computed on crossover latencies did not reveal any reliable differences among the groups $(H=4.77$, $p>.05$ ), whereas the Kruskal-Wallis ANOVA computed on TTW scores indicated significant differences among the PRE, no-PRE, and PA groups $(H=12.17, p<.05)$. Figure 2 presents the median TTW scores for the three groups of rats. As can be seen from the figure, both the PRE and no-PRE groups showed higher preference for the white chamber than did the PA group. These impressions were confirmed by Mann-Whitney $U$ tests, which revealed that both the PRE and the no-PRE groups reliably differed from the PA group $(U=2, p<.01$ and $U=$ $6, p<.01$, respectively). Furthermore, the PRE and noPRE groups did not significantly differ from each other $(U=25, p>.10)$. These results suggest that the swim treatment used in this study was not acting as a punisher in conjunction with the footshock, since preexposing subjects to the swim treatment prior to training did not reduce or attenuate the memory-enhancing effect of the swim treatment.

An ANOVA on the swim time required to reach the criterion of fatigue for rats in the PRE group during the

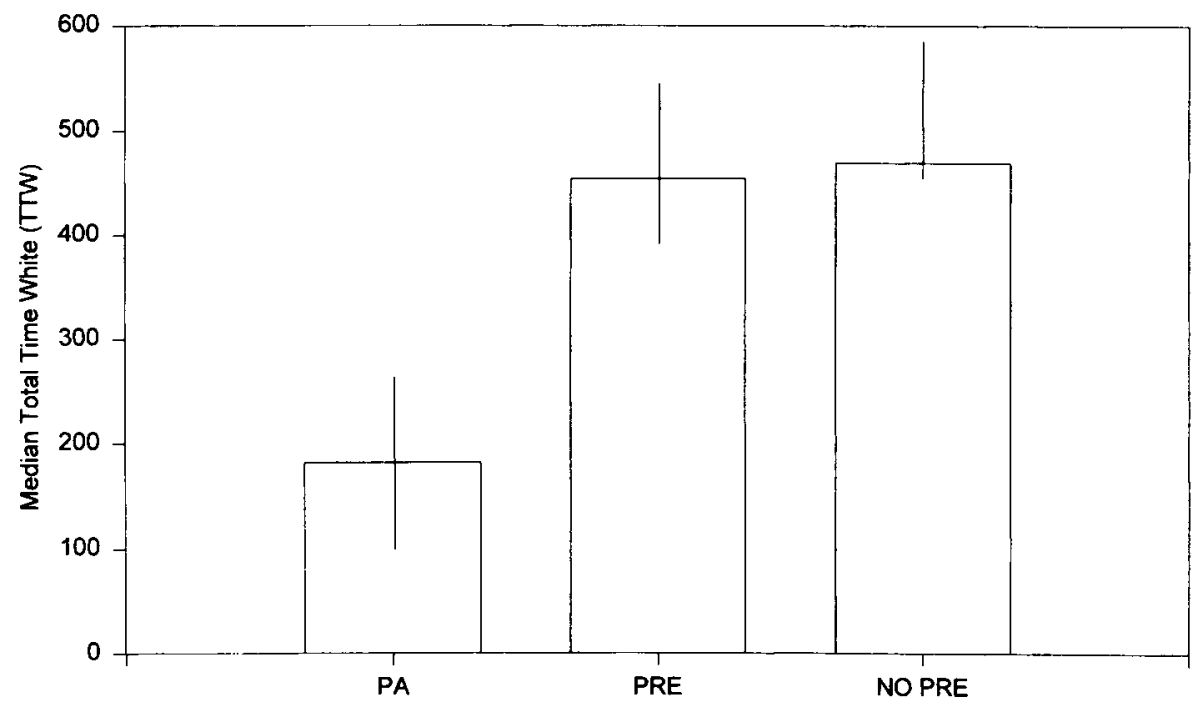

Figure 2. Median TTW scores from the 600-sec retention test for the PA, PRE, and no-PRE groups in Experiment 2. (Error bars = semi-interquartile range.) TTW, total time spent in the white compartment; PA passive avoidance; PRE, preexposure. 
preexposure phase revealed the development of tolerance to the swim treatment, as indicated by reliably longer swimming time required to reach criterion during the preexposure swim treatments $[F(6,42)=3.10, p<$ $.05]$. Furthermore, the swim times of the no-PRE group on the training day and the swim times of the PRE group on the 1st day of preexposure did not reliably differ from each other $(t=1.42, p>.05)$, whereas the swim times between the PRE and no-PRE groups were reliably different on the conditioning day $(t=4.04, p<.01)$. This provides further evidence that the animals in the PRE group became at least partially tolerant to the swim stress. At the same time, however, the tolerance to the swim treatment shown by subjects in the PRE group did not alter the effectiveness of the swim treatment to enhance memory for PA training.

The results of this experiment replicate those of Experiment 1 in that they demonstrate that immediate posttraining exposure to a swim treatment can enhance performance for one-trial inhibitory conditioning in rats. Furthermore, these results indicate that the effectiveness of the swim treatment was not reduced when subjects were exposed to the treatment prior to conditioning, which in turn suggests that the swim treatment is acting as a memory modulator rather than as a punishing agent. The fact that the preexposures occurred in a separate and distinctly different context would seem to support the contention that the training and treatment contexts were resistant to any association.

\section{GENERAL DISCUSSION}

The results of Experiments 1 and 2 suggest that an immediate, posttraining swim treatment can enhance memory for one-trial PA learning. In Experiment 1, rats given an immediate posttraining administration of the swim treatment demonstrated reliably better retention scores for PA training than did groups not exposed to the treatment on a 24-h retention test. Experiment 1 further showed that if the swim treatment was delayed for $15 \mathrm{~min}$, no enhancement of memory occurred, indicating the timedependent nature of swim stress as a memory enhancer. Experiment 2 was designed to test the possibility that the immediate swim treatment was acting as a punisher rather than as a memory modulator. Subjects that were administered seven exposures to the swim treatment prior to training still demonstrated enhanced memory for PA training, suggesting that the swim treatment was serving as a memory modulator rather than as a punisher.

Notable in Experiment 1 was the poor performance of the PA group compared with that of the nonshocked control group. One possibility for the failure to obtain good retention scores in the PA group is that learning took place but did not show up on the retention test. This explanation receives some support from the finding that animals in the PA group from Experiment 2 demonstrated higher TTW scores than did animals in the control (noshock) group in Experiment 1, even though both experiments used the same training intensity $(U=12, p<.05)$.
Although no explicit conditions were included in Experiment 2 to examine learning in the PA group, this crossexperiment comparison suggests that the performance of the PA group in Experiment 1 may not have reflected an absence of learning. Additionally, many studies using inhibitory avoidance conditioning to investigate the memory-modulating properties of various compounds or treatments fail to incorporate a no-shock control group. In these studies, it is argued that the weak learning episode is facilitated by a posttraining treatment; however, no attempt is made to confirm whether or not the control group initially learned the task (e.g., Gold, 1986; Gold \& Van Buskirk, 1975, 1976; Liang, Juler, \& McGaugh, 1986; Sternberg, Martinez, Gold, \& McGaugh, 1985; White \& LeGree, 1984).

It has long been demonstrated that prior experience with a UCS leads to retarded acquisition when that stimulus is later paired with a neutral stimulus -a phenomenon known as the UCS preexposure effect (see Randich, 1981, and Randich \& LoLordo, 1979, for reviews). If the swim treatment had been acting as a punishing agent in our design, preexposure to the swim treatment should have weakened or attenuated its ability to enhance memory. Typically, only a few trials of preexposure to the target stimulus are necessary to retard later conditioning. However, in Experiment 2, seven preexposures to the swim treatment prior to PA conditioning still failed to disrupt the memory-enhancing capabilities of the stress treatment. Since there were many preexposures to the swim treatment and the swimming context, and the data indicated that they failed to attenuate the memory-enhancing effects, it appears that the swim treatment was not having an additive effect with the training shock.

One way to circumvent the problem of the additive punishing effects from the shock and swim treatment would be to apply the swim treatment paradigm to an appetitive task. Recently our lab has investigated the effect of posttraining swim treatment on rats' latency to lick for water in a runway alley maze (Flint $\&$ Riccio, 1995). Preliminary results indicate that swim stress has no effect on behavior $(U=6, p>.05)$. If the swim stress had a punishing effect, the stressed subjects should have had significantly longer latencies to drink on the test day, but the data showed no evidence to support this conclusion.

Some researchers have reported that levels of epinephrine may as much as triple during bouts of intensive exercise (Dimsdale et al., 1984), and others have demonstrated that increasing levels of epinephrine stimulate secretion of blood glucose and ACTH (Ellis et al., 1967). Increased levels of blood glucose in response to stress is a well-established finding (Gold, 1991), and more recently the same results have been demonstrated with both acute (Armario, Castellanos, \& Balasch, 1985) and chronic swim stress (Tan, Morimoto, Sugiura, Morimoto, \& Murakami, 1992). The finding that chronic swimming still induces an increase in circulating blood glucose supports our contention that the enhanced performance by preexposed subjects in Experiment 2 was a result of memory modulation and not punishment. Furthermore, previous 
research has indicated that epinephrine induces secretion of endogenous ACTH (Axelrod \& Reisine, 1984), another hormone that has been reported to enhance memory for a variety of learning tasks (Richardson \& Riccio, 1984). Although endogenous levels of epinephrine, glucose, or $\mathrm{ACTH}$ were not measured in this study, it is plausible that the enhancing effect of the swim treatment is related to the release of one or more of these endogenous compounds.

Although we cannot ascertain the underlying mechanisms of this memory-modulating effect, these results complement other physiological and behavioral evidence suggesting that posttraining stressors can have a memorymodulating function. Furthermore, this study broadens the findings from prior research using shock to include a more naturalistic stressor, swim treatment, that lacks an acute painful component.

\section{REFERENCES}

Armario, A., Castellanos, J. M., \& Balasch, J. (1985). Chronic noise stress and insulin secretion in male rats. Physiology \& Behavior, 34, 359-361.

AXElRoD, J., \& Reisine, T. D. (1984). Stress hormones: Their interaction and regulation. Science, 224, 452-459.

Capaldi, E. D., Sheffer, J. D., Viveiros, D. M., Davidson, D. L., \& CAMPBEll, D. H. (1985). Shock preexposure and the reduced effectiveness of shock. Learning \& Motivation, 16, 357-380.

DE WEID, D. (1980). Hormonal influences on motivation, learning, memory, and psychosis. In D. T. Krieger \& J. C. Hughes (Eds.) Neuroendocrinology (pp. 194-204). Sunderland, MA: Sinauer.

Dimsdale, J. E., Hartley, L. H., Guiney, T., Ruskin, J. N., \& GreenBLAT, D. (1984). Postexercise peril: Plasma catecholamines and exercise. Journal of the American Medical Association, 251, 630-632.

Ellis, S., Kennedy, B. L., Eusebi, A. J., \& Vincent, N. H. (1967) Autonomic control of metabolism. In The physiology of diuretic agents (Annals of the New York Academy of Sciences, Vol. 139, pp. 826-832). New York: New York Academy of Sciences.

Flint, R. W., JR., \& Riccio, D. C. (1995). [Unpublished raw data].

Flint, R. W., JR., \& RICCIO, D. C. (1996). Glucose administration attenuates hypothermia-induced retrograde amnesia in rats in a timeand dose-dependent manner. Psychobiology, 24, 62-66.

GolD, P. E. (1986). Glucose modulation of memory storage processing. Behavioral \& Neural Biology, 45, 342-349

GoLD, P. E. (1991). An integrated memory regulation system: From blood to brain. In R. C. A. Fredrickson, J. L. McGaugh, \& D. L. Felten (Eds.), Peripheral signaling of the brain: Role in neural-immune interactions and learning and memory (pp. 421-441). Toronto: Hogrefe \& Huber.
GOLD, P. E., \& VAN BUSKIRK, R. B. (1975). Facilitation of timedependent memory processes with posttrial epinephrine injections. Behavioral Biology, 13, 145-153.

GOLD, P. E., \& VAN BUSKIRK, R. B. (1976). Enhancement and impairment of memory processes with post-trial injections of adrenocorticotrophic hormone. Behavioral Biology, 16, 387-400.

Harrod, S. B., Metzger, M. M., \& Riccio, D. C. (1996). Does induced recovery from amnesia represent a disinhibition effect? Physiology \& Behavior, 60, 1375-1378.

KumaR, K. B., \& KaRANTH, K. S. (1993). Enhanced processing of an aversive memory following inescapable shock in rats. Biological Psychiatry, 33, 169-172.

Liang, K. C., Juler, R. G., \& McGaugh, J. L. (1986). Modulating effects of posttraining epinephrine on memory: Involvement of the amygdala noradrenergic system. Brain Research, 368, 125-133.

MCGAUGH, J. L. (1989). Involvement of hormonal and neuromodulatory systems in the regulation of memory storage. Annual Review of Neuroscience, 12, 255-287.

Newberry, B. H., Jaikins-Madden, J. E., \& Gerstenberger, T. J. (1991). A holistic conceptualization of stress and disease. New York: AMS Press.

RANDICH, A. (1981). The US preexposure phenomenon in the conditioned suppression paradigm: A role for conditioned situational stimuli. Learning \& Motivation, 12, 321-341.

Randich, A., \& LoLORDO, V. M. (1979). Associative and nonassociative theories of the UCS preexposure phenomenon: Implications for Pavlovian conditioning. Psychological Bulletin, 86, 523-548.

Richardson, R., \& Riccio, D. C. (1984). Memory processes, ACTH, and extinction phenomena. In L. Dachowski \& C. F. Flaherty (Eds.), Current topics in animal learning: Brain, emotion, and cognition (pp. 1-23). London: Erlbaum.

Richardson, R., Riccio, D. C., \& DEvine, L. (1984). ACTH-induced recovery of extinguished avoidance responding. Physiological Psychology, 12, 184-192.

Shors, T. J., Weiss, C., \& Thompson, R. F. (1992). Stress-induced facilitation of classical conditioning. Science, 257, 537-539.

Sternberg, D. B., Martinez, J. L., Gold, P. E., \& McGaugh, J. L. (1985). Age-related memory deficits in rats and mice: Enhancement with peripheral injections of epinephrine. Behavioral \& Neural Biology, 44, 213-220.

Tan, N., Morimoto, K., Sugiura, T., Morimoto, A., \& Murakami, N. (1992). Effects of running training on the blood glucose and lactate in rats during rest and swimming. Physiology \& Behavior, 51 , 927-931.

White, N. M., \& LeGree, P. (1984). Effect of posttraining exposure to an aversive stimulus on retention. Physiological Psychology, 12, 233-236.

(Manuscript received May 21, 1996; revision accepted for publication September 15, 1996.) 